\title{
Editorial Comment on "Enhanced Recovery After Surgery (ERAS) in Head and Neck Oncologic Surgery: A Case-Matched Analysis of Perioperative and Pain Outcomes"
}

\author{
Shuqing Chen, MS, and Baran D. Sumer, MD, FACS \\ Department of Otolaryngology-Head and Neck Surgery, University of Texas Southwestern Medical Center, Dallas, TX
}

Enhanced recovery after surgery (ERAS) programs are multidisciplinary initiatives involving surgeons, anesthesiologists, coordinators, and nursing staff that aim to improve the perioperative patient experience and outcomes in major surgery. ${ }^{1}$ Over the past 2 decades, ERAS programs have been successfully implemented for perioperative care, first in colorectal surgery and then extended to other fields. ${ }^{2-4}$ ERAS protocols call for optimizing elements of care that can significantly decrease perioperative morbidity and mortality. ${ }^{5}$ The principles of ERAS include patient education, goal-directed fluid management, multimodal analgesia, and early mobilization. ${ }^{6}$ Implementing ERAS pathways for major surgical procedures reduces hospital stays and complications, promotes maintenance of an anabolic state for better healing, and decreases reported pain and stress. The international ERAS society has published a series of guidelines with procedurespecific recommendations, however the evidence base for the interventions relevant to head and neck surgery was variable and amalgamated data from a variety of different head and neck cancer procedures. A consensus review recommended that elements relevant to perioperative care in head and neck cancer should be clinically evaluated in a more standardized fashion, highlighting the fact that head and neck surgery has not traveled as far along the ERAS path as other surgical fields. ${ }^{7}$

(C) Society of Surgical Oncology 2020

First Received: 26 October 2020

Accepted: 26 October 2020;

Published Online: 11 November 2020

B. D. Sumer, MD, FACS

e-mail: Baran.sumer@utsouthwestern.edu
After that report, various groups adopted ERAS protocols for major head and neck surgery. ${ }^{8}$ A diverse set of studies with standardized perioperative management strategies have reduced perioperative pain, ${ }^{9}$ shown improved hemodynamic stability and reduction in the inflammatory response, ${ }^{10}$ and reduced hospital length of stay, pain, and opioid use. ${ }^{11}$ While these studies suggest ERAS can improve outcomes in major head and neck surgery, they involve small patient sample sizes, measure single or limited outcomes, endorse limited interventions, and include variable patient populations. A multidisciplinary ERAS protocol implemented prospectively showed the feasibility of developing a standardized and comprehensive protocol for major head and neck surgery ${ }^{6}$ that follows the principles of ERAS. These interventions spanned the preoperative to postoperative and follow-up periods and included tracking outcomes across this timespan.

In this issue of Annals of Surgical Oncology, Kiong et al. use this comprehensive Head and Neck/Reconstructive Surgery ERAS (HNRS-ERAS) pathway and narrow the definition of major head and neck surgery to oncologic surgeries requiring microvascular free-flap reconstruction, since these procedures are complex, involve significant blood loss and postoperative pain, as well as lengthy hospital stays and complex multidisciplinary rehabilitation. ${ }^{12}$

The investigators conducted a case-matched cohort study using the HNRS-ERAS pathway to intervene and track outcomes based on the principles of patient education, goal-directed fluid management, multimodal analgesia, and early mobilization. The outcomes tracked were based on the HNRS National Surgical Quality Improvement Program (NSQIP) database. 
Kiong et al. report that patients receiving ERAS strategies had demographics similar to the matched controls ( $n=200$ in each group); however, the ERAS group had fewer planned intensive care unit (ICU) admissions (4.0\% vs. $14.0 \%$ ), reduced mean length of stay by 1.5 days, and fewer overall complications ( $18.6 \%$ vs. $27.0 \%, p=0.045$ ). A significant reduction in morphine milligram equivalents (MMEs) required over $72 \mathrm{~h}$ was seen in the ERAS group $(138.8 \pm 181.5$ vs. $207.9 \pm 205.5 ; p<0.001){ }^{12}$

This study represents a key addition to the ERAS literature in general and sets the standard for perioperative management for major head and neck surgery requiring free-flap reconstruction. The HNRS-ERAS pathway described should become a cornerstone of managing these complex patients.

DISCLOSURE Shuqing Chen and Baran D. Sumer have no relevant conflicts of interest to report.

\section{REFERENCES}

1. Fearon KC, Ljungqvist O, Von Meyenfeldt M, et al. Enhanced recovery after surgery: a consensus review of clinical care for patients undergoing colonic resection. Clin Nutr. 2005;4:466-77.

2. Melnyk M, Casey RG, Black P, Koupparis AJ. Enhanced recovery after surgery (ERAS) protocols: time to change practice? Can Urol Assoc J. 2011;5:342-8.

3. Kehlet H, Wilmore DW. Multimodal strategies to improve surgical outcome. Am J Surg. 2002;183:630-41.

4. Wind J, Polle SW, Fung Kon Jin PH et al. Systematic review of enhanced recovery programmes in colonic surgery. Br J Surg. 2006;93:800-9.
5. Jones C, Kelliher L, Dickinson M, et al. Randomized clinical trial on enhanced recovery versus standard care following open liver resection. Br J Surg. 2013;100:1015-24.

6. Low GMI, Kiong KL, Amaku R, et al. Feasibility of an Enhanced Recovery After Surgery (ERAS) pathway for major head and neck oncologic surgery. Am J Otolaryngol. 2020;41:102679.

7. Dort JC, Farwell DG, Findlay M, et al. Optimal perioperative care in major head and neck cancer surgery with free flap reconstruction: a consensus review and recommendations from the enhanced recovery after surgery society. JAMA Otolaryngol Head Neck Surg. 2017;143:292-303.

8. Huber GF, Dort JC. Reducing morbidity and complications after major head and neck cancer surgery: the (future) role of enhanced recovery after surgery protocols. Curr Opin Otolaryngol Head Neck Surg. 2018;26:71-7.

9. Townsend M, Liou T, Kallogjeri D, et al. Effect of perioperative gabapentin use on postsurgical pain in patients undergoing head and neck mucosal surgery: a randomized clinical trial. JAMA Otolaryngol Head Neck Surg. 2018;144:959-66.

10. Imai T, Kurosawa K, Yamaguchi K, et al. Enhanced Recovery After Surgery program with dexamethasone administration for major head and neck surgery with free tissue transfer reconstruction: initial institutional experience. Acta Otolaryngol. 2018;138:664-9.

11. Jandali DB, Vaughan D, Eggerstedt M, et al. Enhanced recovery after surgery in head and neck surgery: reduced opioid use and length of stay. Laryngoscope. 2020;130:1227-32.

12. Kiong $\mathrm{KL}, \mathrm{Vu} \mathrm{CN}$, Yao $\mathrm{C}$, et al. Enhanced Recovery After Surgery (ERAS) in head and neck oncologic surgery: a casematched analysis of perioperative and pain outcomes. Ann Surg Oncol. 2020. https://doi.org/10.1245/s10434-020-09174-2

Publisher's Note Springer Nature remains neutral with regard to jurisdictional claims in published maps and institutional affiliations. 